\title{
Proximate Composition, Minerals and Fatty Acid Composition of Juglans Regia L. Genotypes and Cultivars Grown in Turkey
}

\author{
Cemile Yerlikaya $^{1}$, Sevil Yucel $^{2 *},{\text { Ümran } \text { Erturk }^{3} \text { and Mihriban Korukluoğlu }}^{4}$ \\ ${ }^{I}$ Istanbul Technical University; Chemical and Metallurgical Engineering Faculty; Chemical Engineering \\ Department; 34469, Maslak/Istanbul-Turkey. ${ }^{2} Y l l d l z$ Technical University; Chemical and Metallurgical \\ Engineering Faculty; Bioengineering Department; 34210, Davutpaşa/Istanbul-Turkey. ${ }^{3}$ Uludag University; Faculty \\ of Agriculture; Department of Horticulture; Görükle, 16059, Bursa-Turkey. ${ }^{4}$ Uludag University; Faculty of \\ Agriculture; Department of Food Engineering; Görükle, 16059, Bursa-Turkey
}

\begin{abstract}
The proximate composition of eleven walnut (Juglans regia L.) genotypes (28 ŞK 010, 28 ŞK 055, 28 ŞK 041, 28 ŞK 601, 28 ŞK 925, 28 ŞK 028, 28 ŞK 118, 28 ŞK 350, 28 ŞK 930, 28 ŞK 850, 28 ŞK 036) and three walnut cultivars (Şebin, Bilecik, Kaman 1) produced in Turkey were determined. The oil content of the samples ranged from 61.32 to $69.35 \%$, corresponding to an energy value of approximately $710 \mathrm{kcal}$ per $100 \mathrm{~g}$ of kernel. The protein content ranged from 10.58 to $18.19 \%$, and the carbohydrate composition was between 9.05 and $18.92 \%$. The ash content ranged from 1.53 to $1.99 \%$, and the moisture content of the kernels was between 1.91 and $4.48 \%$ the oleic acid content of the oils ranged from 17.90 to $33.35 \%$ of the total fatty acids. The linoleic acid content ranged from 43.15 to $60.20 \%$. The linolenic acid content ranged from 9.98 to $13.00 \%$. The palmitic acid content was between 5.21 and $8.40 \%$. Stearic acid ranged from 2.36 to $4.25 \%$. Potassium was the major mineral in all the samples, ranging from 359.73 to $482.97 \mathrm{mg} / 100 \mathrm{~g}$. Calcium was the next most abundant mineral, ranging from 109.45 to $335.97 \mathrm{mg} / 100 \mathrm{~g}$, followed by magnesium, ranging from 126.01 to $165.15 \mathrm{mg} / 100 \mathrm{~g}$.
\end{abstract}

Key words: Juglans regia L., walnut, proximate composition, fatty acid

\section{INTRODUCTION}

The high nutritive value and characteristic healthpromoting components of walnuts make them an indispensable part of the Mediterranean diet. The lipids (tocopherol, fatty acid), protein and minerals contained in the walnuts have beneficial effects on human health. The walnut kernel (Juglans regia L.) generally contains approximately $60 \%$ oil (Prasad 1994). However, this can vary from 52 to $70 \%$. The amount of oil in the walnut kernel depends on the cultivar, location at which grown and the irrigation rate (Greve et al. 1992; Garcia et al. 1994; Çağlarırmak 2003; Akca et al. 2005). The fatty acids present in the walnut oil are primarily unsaturated fatty acids. Compared with most other nuts, which contain mostly monounsaturated fatty acids (MUFA), walnuts are highly enriched in omega- 6 and omega-3 polyunsaturated fatty acids (PUFA). These PUFA are essential dietary fatty acids (Amaral et al. 2003). It has been suggested that the high PUFA content of walnuts can serve to reduce the risk of heart disease. The major fatty acids found in walnuts are linoleic (18:2 w6), $\alpha$ -

*Author for correspondence: yuce.sevil@gmail.com 
linolenic (18:3 w3), oleic (18:1 w9), palmitic (16:0), and stearic (18:0) acid (Ruggeri et al. 1998; Lavedrine et al. 2000; Zwarts et al. 1999; Li et al. 2007). The fatty acid composition of the nut is important to its economic and nutritional value.

In addition to oil, walnuts provide appreciable amounts of proteins (12-24\%), carbohydrates (12$18 \%)$, fibre $(1.5-2 \%)$ and minerals $(1.7-2 \%)$ (Lavedrine et al. 2000; Savage 2001; Amaral et al. 2003; Çağlarırmak 2003; Ozkan and Koyuncu 2005; Akca et al. 2005). Walnuts contain high levels of potassium (390-700 $\mathrm{mg} / 100 \mathrm{~g}$ ), phosphorus (310-510 mg/100 g), and magnesium $(90-140 \mathrm{mg} / 100 \mathrm{~g})$ and have low sodium (1-15 $\mathrm{mg} / 100 \mathrm{~g}$ ) (Souci et al. 1994; Lavedrine et al. 2000; Savage 2001; Akca et al. 2005). Turkey is the motherland of the walnut (Juglans regia L.) and one of the world's oldest areas of walnut cultivation. Turkey has 4.5 million natural seedling hybrid walnut trees. The climatic diversity of the country and the high heterozygosity of the trees as a result of continuing sexual propagation have produced a local walnut population that contains very rich genetic material (Akca and Ozongun 2004). The most promising genotypes are selected for the national germplasm collections. Initially, the goals of walnut breeding efforts were blight resistance and anthracnose tolerance, high-quality fruit and large yields. Other studies have bred for the morphological features that include nut weight, nut length, kernel weight, kernel percentage, shell thickness, kernel colour, kernel fullness, late leafing, and lateral fruitfulness (Olez, 1971; Şen 1983; Celebioglu et al. 1988; Yarılgaç et al. 2001; Akca and Ozongun 2004; Balta et al. 2007). However, studies are also needed to describe the nutritional value of selected varieties. More detailed research on walnuts' nutritional quality and health-promoting components will enhance knowledge of the crop and encourage walnut consumption.

The objective of this study was the characterisation of the fatty acid composition of walnut oil of the selected walnut genotypes grown in Şebinkarahisar and of the commonly grown cultivars in Turkey. The genotypes and cultivars were chosen according to their properties and to common quality criteria for the nuts, such as nut weight, nut length, kernel weight, kernel percentage, shell thickness, kernel colour and kernel fullness. The nutrients present in walnuts were analysed to determine their nutritional value.
This information can serve as a resource for assessment by nutritionists, breeders and growers.

\section{MATERIALS AND METHODS}

\section{Walnut samples}

Eleven walnut genotypes (28 ŞK 010, 28 ŞK 055, 28 ŞK 041, 28 ŞK 601, 28 ŞK 925, 28 ŞK 028, 28 ŞK 118, 28 ŞK 350, 28 ȘK 930, 28 ȘK 850, 28 ŞK 036) and three commercial cultivars (Şebin, Bilecik and Kaman 1) were analysed. The walnut fruits of genotypes were collected from Şebinkarahisar province, and the fruits of cultivars were collected from a commercial plantation in Tokat, in the middle Black Sea Region of Turkey. The walnut fruits were harvested in September during the 2005 crop year. After the harvest, the walnut fruits were immediately dried and stored in the shell at room temperature until the start of the analysis.

\section{Chemical analysis}

The dry matter content was determined by drying the samples overnight in a hot-air oven at $105{ }^{\circ} \mathrm{C}$. Ash analysis was carried out by heating the sample in a muffle furnace at $525{ }^{\circ} \mathrm{C}$ for $8 \mathrm{~h}$. The quantity of total protein was calculated by multiplying the nitrogen content, determined using the Kjeldahl method, by the coefficient 6.25 (AOAC, 1990). The carbohydrate content was estimated by calculating the differences based on the other components and using the following formula: Carbohydrate content $(\%)=100 \%-($ moisture $(\%)$ $+\operatorname{protein}(\%)+\operatorname{oil}(\%)+\operatorname{ash}(\%))$ (Grosso et al. 2000). To determine the oil content, the walnuts were ground in a coffee mill for 30 seconds. Immediately after grinding, the walnuts were extracted for $6 \mathrm{~h}$ with hexane using a Soxhlet apparatus (Paquat and Houtfenne 1987). Energy was expressed in kilocalories, using the following formula: Energy $\mathrm{kcal}=4 \mathrm{x}$ (protein $\mathrm{g}+$ carbohydrate g) +9 x (lipid g) (Pereira et al., 2008). The protein, carbohydrate and total oil content of fruits were calculated on a dry weight basis. All the analyses were carried out in triplicate.

\section{Determination of mineral composition}

To determine the mineral composition, 0.3-0.4 g of previously dried, ground and homogenised kernel was placed in a platinum crucible. The 
sample was heated in a muffle furnace at $550{ }^{\circ} \mathrm{C}$ for $6 \mathrm{~h}$. Before this, the sample was partially dissolved in $2 \mathrm{~mL} \mathrm{HNO}_{3}(65 \%)$ and heated on a hot plate until dry to prevent loss from the sample and black smoke during the ash formation. After a 10-min cooling period, the ash was dissolved in 2 $\mathrm{mL} \mathrm{HNO}_{3}(65 \%)$ and diluted with deionised water to a volume of $25 \mathrm{~mL}$. A Unicam flame atomic absorption instrument (Model 929 AA) was used for the determination of $\mathrm{Na}, \mathrm{Mg}, \mathrm{K}, \mathrm{Ca}, \mathrm{Cu}, \mathrm{Zn}$ and $\mathrm{Fe}$ in the sample digests. The results were expressed on a dry-matter basis. All the mineral composition analyses were performed in triplicate.

\section{Determination of fatty acid composition}

The fatty acid composition of the walnut samples was determined by the gas chromatography (GC). The oil samples obtained via Soxhlet extraction were converted to their corresponding methyl esters according to the AOCS method (AOCS, 1993). The $\mathrm{BF}_{3} /$ methanol method was used for methylation. The GC analysis of the fatty acid methyl esters (FAME) was performed using a ThermoQuest CE Instrumental Trace 2000 Series GC equipped with a BPX70 Forte Capillary GC column $(60 \mathrm{~m} \times 0.32 \mathrm{~mm} \times 0.25 \mu \mathrm{m}$ film thickness of 5\% biphenyl and $95 \%$ dimethyl polysiloxane). A split injector (split ratio 13:1) and FID detector were used during the analyses. The column temperature programme was $2 \mathrm{~min}$ at $60^{\circ} \mathrm{C}$, $30^{\circ} \mathrm{C} / \mathrm{min}$ to $150^{\circ} \mathrm{C}, 1^{\circ} \mathrm{C} / \mathrm{min}$ to $190^{\circ} \mathrm{C}, 20^{\circ} \mathrm{C} / \mathrm{min}$ to $220^{\circ} \mathrm{C}$ and $10 \mathrm{~min}$ at $220^{\circ} \mathrm{C}$. The injector and detector temperatures were 225 and $250^{\circ} \mathrm{C}$, respectively. The carrier gas was nitrogen at a flow rate of $30 \mathrm{~mL} / \mathrm{min}$. The air and hydrogen flow rates were 350 and $35 \mathrm{~mL} / \mathrm{min}$, respectively. The peaks of fatty acids were identified by comparing the retention times with those of a mixture of isomers of standard methyl esters (Sigma Chemicals Co. Deisenhofen, Germany). All the statistical analyses were performed in triplicate, and the data were expressed as averages \pm standard deviations (SD).

\section{RESULTS AND DISCUSSION}

The chemical compositions of the cultivars and genotypes are shown in Table 1. Oil was the predominant component, ranging from 61.32 to $69.35 \%$, followed by carbohydrates $(9.05-18.92$ $\%)$ and protein (10.58-18.19\%). The moisture content of the kernels ranged between 1.91-4.48\%.
Total ash ranged between 1.53-1.99\%. In general, these findings agreed with the previously published values. Şahin and Akbaş (2001) have reported that 35 walnut genotypes from Turkey contained $56.38-70.59 \%$ oil, $13.59-22.99 \%$ protein, and 1.66-2.81\% ash. Ozkan and Koyuncu (2005) have determined the chemical composition of 10 walnut genotypes as follows: oil, 61.97$74.95 \%$; carbohydrate, $8.05-13.23 \%$; protein 15.17-19.24\%; and ash, 1.26-2.06\%. Pereira et al. (2008) have evaluated the chemical composition of six walnut cultivars in Portugal. They found that the total oil content of the nuts ranged from 68.83 to $72.14 \%$. The protein content ranged from 14.38 to $18.03 \%$, and the carbohydrate content ranged from 3.75 to $6.10 \%$. The genotypes and cultivars analysed in the current study had a higher carbohydrate content than the cultivars reported in the literature. These differences could be attributed to the year of harvest and the accompanying environmental conditions. Indeed, different values of temperature, rainfall and light can influence the chemical composition of fruits. The energy values determined in the current study ranged from 682 to $728 \mathrm{kcal}$ and were similar to the values reported by Amaral et al. (2003) and Pereira et al. (2008).

The mineral composition of the genotypes and cultivars studied is shown in Table 2. The genotypes and cultivars contained varying amounts of $\mathrm{K}, \mathrm{Ca}, \mathrm{Mg}, \mathrm{Na}, \mathrm{Mn}, \mathrm{Fe}, \mathrm{Cu}$ and $\mathrm{Zn}$. The values found were $(\mathrm{mg} / 100 \mathrm{~g}): 359.73-482.99$, $109.45-335.97,126.01-165.15,2.45-9.99,1.52-$ $4.79, \quad 2.78-4.85, \quad 0.92-1.80$, and 2.45-4.30, respectively. High levels of the major minerals potassium, calcium and magnesium were found. Very low levels of sodium were detected. Genotypes had the most marked effects on the potassium, calcium and sodium concentrations. For example, 28 SSK 118 walnuts contained the lowest level $(109.45 \mathrm{mg} / 100 \mathrm{~g})$ of calcium found, whereas 28 ŞK 601 had the highest level (335.97 $\mathrm{mg} / 100 \mathrm{~g}$ ). Similar results were observed for the potassium and sodium. However, these differences were less pronounced than those found for the calcium levels of the different genotypes. The Kaman 1 cultivar exhibited significant differences in the form of low calcium $(149.32 \mathrm{mg} / 100 \mathrm{~g})$ and magnesium $(126.95 \mathrm{mg} / 100 \mathrm{~g})$ levels. However, it had the highest sodium $(9.99 \mathrm{mg} / 100 \mathrm{~g})$ and manganese $(4.79 \mathrm{mg} / 100 \mathrm{~g})$ values of all the cultivars analysed. The mineral compositions of two walnut cultivars (Franquette and Hartley) originating in France and in California have been 
determined by Lavedrine et al. (2000). The potassium levels of the French walnuts were higher (Franquette: 487 and Hartley: 466 mg/100 g) than those of the California walnuts (Franquette: 358 and Hartley: $372 \mathrm{mg} / 100 \mathrm{~g}$ ). Inversely, the sodium level was higher in the Californian Hartley than in the French Hartley $(6.7-0.3 \mathrm{mg} / 100 \mathrm{~g})$. Akca et al. (2005) have also compared the mineral composition of Turkish walnut cultivars. The amounts of minerals found by that study $(\mathrm{mg} / 100 \mathrm{~g})$ ranged between $170-419$ for potassium, 102-146 for magnesium, 202-453 for calcium, 0.4-3.5 for sodium, 1.2-6.0 for manganese, 1.2-6.9 for iron, 1.2-3.8 for zinc and 0.5-2.2 for copper. The elemental composition and the $\mathrm{pH}$ of the soil are well known to have a great influence on the absorption of minerals by plants (Charlot and Germain 1994; Korzeniowska and Stanislawska-Glubiak 2004).

Table 1 - Proximate Composition of Eleven Walnut Genotypes and Three Commercial Cultivars Grown in Turkey*.

\begin{tabular}{lccccccc}
\hline $\begin{array}{c}\text { Genotypes } \\
\text { and Cultivars }\end{array}$ & $\begin{array}{c}\text { Dry Matter } \\
(\boldsymbol{\%})\end{array}$ & $\begin{array}{c}\text { Moisture } \\
(\boldsymbol{\%})\end{array}$ & $\begin{array}{c}\text { Total Oil } \\
(\boldsymbol{\%})\end{array}$ & $\begin{array}{c}\text { Protein } \\
(\boldsymbol{\%})\end{array}$ & $\begin{array}{c}\text { Carbohydrate } \\
(\boldsymbol{\%})\end{array}$ & $\begin{array}{c}\text { Ash } \\
(\boldsymbol{\%})\end{array}$ & $\begin{array}{c}\text { Energy } \\
(\mathbf{k c a l})\end{array}$ \\
\hline 28 ŞK 010 & $98.09 \pm 0.82$ & $1.91 \pm 0.82$ & $66.67 \pm 1.19$ & $11.56 \pm 1.20$ & $18.28 \pm 1.83$ & $1.58 \pm 0.27$ & $719 \pm 3.38$ \\
28 ŞK 055 & $95.52 \pm 0.71$ & $4.48 \pm 0.71$ & $65.69 \pm 1.14$ & $13.78 \pm 0.69$ & $14.38 \pm 2.02$ & $1.67 \pm 0.26$ & $704 \pm 6.78$ \\
28 ŞK 041 & $95.64 \pm 1.00$ & $4.36 \pm 1.00$ & $67.07 \pm 1.22$ & $12.40 \pm 0.56$ & $14.39 \pm 0.70$ & $1.78 \pm 0.13$ & $711 \pm 10.13$ \\
28 ŞK 601 & $95.53 \pm 2.13$ & $4.47 \pm 2.13$ & $64.84 \pm 1.66$ & $10.91 \pm 2.86$ & $18.02 \pm 3.22$ & $1.76 \pm 0.23$ & $699 \pm 16.97$ \\
28 ŞK 925 & $96.01 \pm 1.00$ & $3.99 \pm 1.00$ & $68.40 \pm 1.25$ & $12.73 \pm 2.61$ & $13.00 \pm 3.51$ & $1.88 \pm 0.49$ & $718 \pm 2.54$ \\
28 ŞK 028 & $96.54 \pm 2.34$ & $3.46 \pm 2.34$ & $66.09 \pm 1.97$ & $12.52 \pm 1.86$ & $16.15 \pm 1.44$ & $1.78 \pm 0.34$ & $709 \pm 19.24$ \\
28 ŞK 118 & $96.66 \pm 2.30$ & $3.34 \pm 2.30$ & $68.59 \pm 2.14$ & $11.78 \pm 1.18$ & $14.35 \pm 3.78$ & $1.94 \pm 0.49$ & $722 \pm 7.18$ \\
28 ŞK 350 & $96.44 \pm 1.64$ & $3.56 \pm 1.64$ & $64.60 \pm 1.39$ & $12.64 \pm 1.19$ & $17.42 \pm 1.16$ & $1.78 \pm 0.42$ & $702 \pm 13.31$ \\
28 ŞK 930 & $96.81 \pm 0.45$ & $3.19 \pm 0.45$ & $66.33 \pm 1.12$ & $15.50 \pm 0.56$ & $13.08 \pm 1.07$ & $1.90 \pm 0.65$ & $711 \pm 7.97$ \\
28 ŞK 850 & $96.56 \pm 1.37$ & $3.44 \pm 1.37$ & $65.92 \pm 1.30$ & $12.95 \pm 0.97$ & $15.75 \pm 1.53$ & $1.94 \pm 0.86$ & $708 \pm 13.09$ \\
28 ŞK 036 & $95.81 \pm 1.92$ & $4.19 \pm 1.92$ & $61.32 \pm 0.93$ & $13.58 \pm 0.50$ & $18.92 \pm 3.01$ & $1.99 \pm 0.78$ & $682 \pm 9.10$ \\
Şebin & $97.05 \pm 0.39$ & $2.95 \pm 0.39$ & $69.35 \pm 1.05$ & $14.00 \pm 0.43$ & $11.95 \pm 1.51$ & $1.75 \pm 0.75$ & $728 \pm 6.95$ \\
Bilecik & $96.30 \pm 1.13$ & $3.70 \pm 1.13$ & $65.79 \pm 2.39$ & $10.58 \pm 0.80$ & $18.40 \pm 2.26$ & $1.53 \pm 0.51$ & $708 \pm 14.67$ \\
Kaman 1 & $96.07 \pm 1.11$ & $3.90 \pm 1.11$ & $67.13 \pm 0,73$ & $18.19 \pm 1.23$ & $9.05 \pm 2.74$ & $1.73 \pm 0.75$ & $713 \pm 3.52$ \\
\hline
\end{tabular}

* Results are the mean value \pm standard deviation of three independent experiments.

Table 2 - Same Minerals Compositions Contents of Genotypes and Cultivars*

\begin{tabular}{lllllllll}
\hline $\begin{array}{c}\text { Genotypes and } \\
\text { Cultivars }\end{array}$ & \multicolumn{1}{c}{$\mathbf{K}$} & \multicolumn{1}{c}{$\mathbf{C a}$} & \multicolumn{1}{c}{$\mathbf{M g}$} & $\mathbf{N a}$ & $\mathbf{M n}$ & $\mathbf{F e}$ & $\mathbf{C u}$ & $\mathbf{Z n}$ \\
\hline 28 ŞK 010 & $417.33 \pm 12.6$ & $163.19 \pm 7.5$ & $142.12 \pm 4.4$ & $7.16 \pm 1.0$ & $3.47 \pm 0.8$ & $4.05 \pm 0.4$ & $1.24 \pm 0.2$ & $2.45 \pm 0.5$ \\
28 ŞK 055 & $424.05 \pm 12.1$ & $165.82 \pm 8.4$ & $139.24 \pm 9.0$ & $4.99 \pm 1.7$ & $2.63 \pm 0.5$ & $4.85 \pm 0.6$ & $1.49 \pm 0.4$ & $3.75 \pm 0.5$ \\
28 ŞK 041 & $385.33 \pm 14.7$ & $218.62 \pm 14.1$ & $126.01 \pm 8.5$ & $4.98 \pm 2.1$ & $1.52 \pm 0.4$ & $3.98 \pm 1.4$ & $1.22 \pm 0.2$ & $3.8 \pm 0.5$ \\
28 ŞK 601 & $451.38 \pm 13.0$ & $335.97 \pm 14.5$ & $147.89 \pm 15.3$ & $8.17 \pm 1.9$ & $2.98 \pm 0.8$ & $3.67 \pm 0.4$ & $1.80 \pm 0.2$ & $4.30 \pm 0.8$ \\
28 ŞK 925 & $463.91 \pm 8.1$ & $147.62 \pm 11.1$ & $136.96 \pm 15.4$ & $5.84 \pm 2.1$ & $4.28 \pm 0.6$ & $3.33 \pm 0.4$ & $1.13 \pm 0.1$ & $3.17 \pm 0.4$ \\
28 ŞK 028 & $368.48 \pm 18.4$ & $216.35 \pm 23.5$ & $138.27 \pm 14.9$ & $2.45 \pm 1.2$ & $2.44 \pm 0.7$ & $3.18 \pm 0.3$ & $1.63 \pm 0.4$ & $3.43 \pm 0.3$ \\
28 ŞK 118 & $476.99 \pm 15.2$ & $109.45 \pm 12.2$ & $127.01 \pm 11.3$ & $2.55 \pm 1.3$ & $3.57 \pm 0.4$ & $2.78 \pm 0.4$ & $1.22 \pm 0.2$ & $2.72 \pm 0.7$ \\
28 ŞK 350 & $470.80 \pm 11.0$ & $121.20 \pm 18.0$ & $133.05 \pm 11.0$ & $3.77 \pm 1.3$ & $2.02 \pm 0.2$ & $3.19 \pm 0.4$ & $0.92 \pm 0.2$ & $2.90 \pm 0.4$ \\
28 ŞK 930 & $482.97 \pm 12.4$ & $161.97 \pm 7.2$ & $137.88 \pm 15.4$ & $2.45 \pm 0.9$ & $3.97 \pm 0.4$ & $3.84 \pm 0.3$ & $1.10 \pm 0.2$ & $3.86 \pm 0.4$ \\
28 ŞK 850 & $441.88 \pm 13.7$ & $164.66 \pm 6.8$ & $142.91 \pm 12.9$ & $2.70 \pm 0.8$ & $3.60 \pm 0.6$ & $3.31 \pm 0.5$ & $1.58 \pm 0.3$ & $3.63 \pm 0.5$ \\
28 ŞK 036 & $417.13 \pm 16.9$ & $156.77 \pm 7.8$ & $126.86 \pm 15.3$ & $3.05 \pm 0.9$ & $1.60 \pm 0.3$ & $3.22 \pm 0.6$ & $1.38 \pm 0.3$ & $3.43 \pm 0.5$ \\
Şebin & $359.73 \pm 15.2$ & $200.39 \pm 9.0$ & $156.33 \pm 17.3$ & $5.43 \pm 0.6$ & $4.32 \pm 0.6$ & $3.68 \pm 0.5$ & $1.55 \pm 0.4$ & $2.94 \pm 0.3$ \\
Bilecik & $389.20 \pm 10.2$ & $202.26 \pm 14.5$ & $165.15 \pm 15.9$ & $6.46 \pm 1.2$ & $3.97 \pm 0.9$ & $3.58 \pm 0.5$ & $1.57 \pm 0.4$ & $2.99 \pm 0.4$ \\
Kaman 1 & $366.81 \pm 6.7$ & $149.32 \pm 18.1$ & $126.95 \pm 13.3$ & $9.99 \pm 1.2$ & $4.79 \pm 0.8$ & $3.27 \pm 0.5$ & $1.54 \pm 0.7$ & $3.48 \pm 0.5$ \\
\hline
\end{tabular}

*Results are the mean value \pm standard deviation of three independent experiments as $\mathrm{mg} / 100 \mathrm{~g}$

The results for the fatty acid composition, total saturated fatty acids (SFA), monounsaturated fatty acids (MUFA) and polyunsaturated fatty acids
(PUFA) for the walnuts analysed are shown in Table 3. The major fatty acids in the Turkish samples were linoleic (C18:2), followed by oleic 
(C18:1w9), $\alpha$-linolenic (C18:3), palmitic (C16:0) and stearic acid (C18:0). Linoleic acid was the most abundant fatty acid in all the walnuts analysed, with values ranging from $43.15 \%$ in 28 ŞK 925 to $60.20 \%$ in 28 ŞK 055 . Oleic acid, the second in order of importance, ranged from $17.90 \%$ (28 ŞK 850 ) to $33.5 \%$ (28 ŞK 925), followed by linolenic acid, from $9.98 \%$ (28 ŞK
930) to $13.00 \%$ (28 ŞK 041 and 28 ŞK 850 ). Of the remaining fatty acids, only palmitic (5.21$8.40 \%)$ and stearic acid (2.36-4.25\%) were present in considerable amounts. The main fatty acids occurred in amounts generally similar to those already described for walnuts grown in Turkey (Çağlarırmak 2003; Ozkan and Koyuncu 2005; Pereira et al. 2008).

Table 3 - Fatty Acid Composition Content (mg/g) of Oil Extracted From Analyzed Walnut Genotypes and Cultivars*

\begin{tabular}{|c|c|c|c|c|c|c|c|c|c|c|c|c|c|}
\hline $\begin{array}{l}\text { Fatty } \\
\text { Acid }\end{array}$ & $\begin{array}{c}28 \text { ŞK } \\
010\end{array}$ & $\begin{array}{c}28 \text { ŞK } \\
055\end{array}$ & $\begin{array}{c}28 \text { ŞK } \\
041\end{array}$ & $\begin{array}{c}28 \text { ŞK } \\
925\end{array}$ & $\begin{array}{c}28 \text { ŞK } \\
028\end{array}$ & $\begin{array}{c}28 \text { ŞK } \\
118\end{array}$ & $\begin{array}{c}28 \text { ŞK } \\
350\end{array}$ & $\begin{array}{c}28 \text { ŞK } \\
930\end{array}$ & $\begin{array}{c}28 \text { ŞK } \\
850\end{array}$ & $\begin{array}{c}28 \text { ŞK } \\
036\end{array}$ & Şebin & Bilecik & $\begin{array}{c}\text { Kaman } \\
\text { I }\end{array}$ \\
\hline C16:0 & $\begin{array}{c}6.26 \\
\pm 0.21\end{array}$ & .32 & $\begin{array}{c}7.33 \\
\pm 0.31\end{array}$ & $\begin{array}{c}7.67 \\
\pm 0.40\end{array}$ & $\begin{array}{c}5.75 \\
\pm 0.15\end{array}$ & $\begin{array}{c}5.21 \\
\pm 0.38\end{array}$ & $\begin{array}{c}7.57 \\
\pm 0.25\end{array}$ & $\begin{array}{c}5.51 \\
\pm 0.10\end{array}$ & $\begin{array}{c}6.29 \pm 0 \\
1\end{array}$ & $\begin{array}{c}6.17 \\
\pm 0.26\end{array}$ & $\begin{array}{c}6.84 \pm 0 \\
17\end{array}$ & $\begin{array}{c}8.40 \\
\pm 0.25\end{array}$ & $\begin{array}{c}7.68 \\
\pm 0.21\end{array}$ \\
\hline C16:01 & $\begin{array}{c}0.13 \\
\pm 0.02\end{array}$ & $\begin{array}{c}0.14 \\
\pm 0.03\end{array}$ & $\begin{array}{c}0.11 \\
\pm 0.01\end{array}$ & $\begin{array}{c}0.13 \\
\pm 0.04\end{array}$ & $\begin{array}{c}0.12 \\
\pm 0.03\end{array}$ & $\begin{array}{c}0.10 \\
\pm 0.01\end{array}$ & $\begin{array}{l}0.10 \\
\pm 0.0\end{array}$ & $\begin{array}{l}0.15 \\
\pm 0.0\end{array}$ & $\begin{array}{c}0.12 \\
\pm 0.01\end{array}$ & $\begin{array}{c}0.20 \\
\pm 0.05\end{array}$ & & $\begin{array}{c}0.23 \\
\pm 0.04\end{array}$ & $\begin{array}{c}0.12 \\
\pm 0.02\end{array}$ \\
\hline C18:0 & $\begin{array}{c}3.22 \\
\pm 0.01\end{array}$ & $\begin{array}{c}2.73 \\
\pm 0.02\end{array}$ & $\begin{array}{c}2.82 \\
\pm 0.09\end{array}$ & $\begin{array}{c}4.25 \\
\pm 0.11\end{array}$ & $\begin{array}{c}2.87 \\
\pm 0.08\end{array}$ & $\begin{array}{c}3.61 \\
\pm 0.20\end{array}$ & $\begin{array}{c}3.00 \\
\pm 0.07\end{array}$ & $\begin{array}{c}3.49 \\
\pm 0.10\end{array}$ & $\begin{array}{c}3.36 \\
\pm 0.18\end{array}$ & $\begin{array}{c}2.49 \\
\pm 0.12\end{array}$ & $\begin{array}{c}2.80 \\
\pm 0.10\end{array}$ & $\begin{array}{c}3.22 \\
\pm 0.11\end{array}$ & $\begin{array}{c}2.36 \\
\pm 0.30\end{array}$ \\
\hline $\begin{array}{l}\text { C18: } \\
1 w 9\end{array}$ & $\begin{array}{l}21.92 \\
\pm 0.32\end{array}$ & $\begin{array}{l}19.03 \\
\pm 0.20\end{array}$ & $\begin{array}{l}21.90 \\
\pm 0.21\end{array}$ & $\begin{array}{l}33.35 \\
\pm 0.40\end{array}$ & $\begin{array}{l}23.80 \\
\pm 1.30\end{array}$ & $\begin{array}{l}30.30 \\
\pm 1.21\end{array}$ & $\begin{array}{l}21.84 \\
\pm 1.53\end{array}$ & $\begin{array}{l}29.37 \\
\pm 0.98\end{array}$ & $\begin{array}{l}17.90 \\
\pm 0.25\end{array}$ & $\begin{array}{l}22.02 \\
\pm 0.61\end{array}$ & & $\begin{array}{l}27.85 \\
\pm 1.25\end{array}$ & $\begin{array}{l}21.84 \\
\pm 1.12\end{array}$ \\
\hline $\begin{array}{l}\text { C18: } \\
1 w 7\end{array}$ & $\mathrm{nd}^{* *}$ & $\begin{array}{c}0.84 \\
\pm 0.02\end{array}$ & $\begin{array}{c}0.83 \\
\pm 0.05\end{array}$ & $\begin{array}{c}0.91 \\
\pm 0.10\end{array}$ & $\begin{array}{c}0.66 \\
\pm 0.06\end{array}$ & $\begin{array}{c}0.51 \\
\pm 0.02\end{array}$ & $\begin{array}{c}0.81 \\
\pm 0.02\end{array}$ & $\begin{array}{c}0.73 \\
\pm 0.05\end{array}$ & $\mathrm{nd}^{* *}$ & $\begin{array}{c}0.43 \\
\pm 0.06\end{array}$ & $\begin{array}{c}0.55 \\
\pm 0.03\end{array}$ & $\begin{array}{c}1.00 \\
\pm 0.06\end{array}$ & $\begin{array}{c}0.93 \\
\pm 0.04\end{array}$ \\
\hline C18:2 & $\begin{array}{l}55.68 \\
\pm 1.50\end{array}$ & $\begin{array}{l}60.20 \\
\pm 0.90\end{array}$ & $\begin{array}{l}53.70 \\
\pm 2.30\end{array}$ & $\begin{array}{l}43.15 \\
\pm 1.40\end{array}$ & $\begin{array}{l}56.30 \\
\pm 2.00\end{array}$ & $\begin{array}{c}47.4 \\
\pm 2.65\end{array}$ & $\begin{array}{l}53.21 \\
\pm 1.73\end{array}$ & $\begin{array}{l}50.44 \\
\pm 1.00\end{array}$ & $\begin{array}{l}59.10 \\
\pm 1.52\end{array}$ & $\begin{array}{l}56.23 \\
\pm 2.06\end{array}$ & $\begin{array}{l}57.80 \\
\pm 1.79\end{array}$ & $\begin{array}{l}47.26 \\
\pm 1.76\end{array}$ & $\begin{array}{l}54.75 \\
\pm 0.98\end{array}$ \\
\hline C18:3 & $\begin{array}{l}12.44 \\
\pm 1.22\end{array}$ & $\begin{array}{l}10.10 \\
\pm 1.13\end{array}$ & $\begin{array}{l}13.00 \\
\pm 0.75\end{array}$ & $\begin{array}{l}10.18 \\
\pm 0.28\end{array}$ & $\begin{array}{l}10.10 \\
\pm 0.39\end{array}$ & $\begin{array}{c}12.5 \\
\pm 0.92\end{array}$ & $\begin{array}{l}13.12 \\
\pm 0.78\end{array}$ & $\begin{array}{c}9.98 \\
\pm 1.20\end{array}$ & $\begin{array}{l}13.00 \\
\pm 1.09\end{array}$ & $\begin{array}{l}12.16 \\
\pm 1.15\end{array}$ & & & $\begin{array}{l}12.01 \\
\pm 0.92\end{array}$ \\
\hline C20:0 & $\begin{array}{c}0.12 \\
\pm 0.05\end{array}$ & $\begin{array}{c}0.10 \\
\pm 0.05\end{array}$ & $\begin{array}{c}0.10 \\
\pm 0.01\end{array}$ & $\begin{array}{c}0.13 \\
\pm 0.01\end{array}$ & $\begin{array}{c}0.10 \\
\pm 0.03\end{array}$ & $\begin{array}{c}0.13 \\
\pm 0.01\end{array}$ & $\begin{array}{c}0.10 \\
\pm 0.01\end{array}$ & $\begin{array}{c}0.23 \\
\pm 0.02\end{array}$ & $\begin{array}{c}0.10 \\
\pm 0.03\end{array}$ & $\begin{array}{c}0.11 \\
\pm 0.02\end{array}$ & & $\begin{array}{c}0.10 \\
\pm 0.01\end{array}$ & $\begin{array}{c}0.10 \\
\pm 0.03\end{array}$ \\
\hline C20: 1 & $\begin{array}{l}0.23 \\
\pm 0.0\end{array}$ & $\begin{array}{c}0.20 \\
\pm 0.02\end{array}$ & $\begin{array}{c}0.22 \\
\pm 0.01\end{array}$ & $\begin{array}{c}0.23 \\
\pm 0.01\end{array}$ & $\begin{array}{c}0.26 \\
\pm 0.01\end{array}$ & $\begin{array}{c}0.25 \\
\pm 0.03\end{array}$ & $\begin{array}{c}0.25 \\
\pm 0.02\end{array}$ & $\begin{array}{l}0.12 \\
\pm 0.0\end{array}$ & $\begin{array}{c}0.13 \\
\pm 0.04\end{array}$ & $\begin{array}{c}0.18 \\
\pm 0.03\end{array}$ & $\begin{array}{c}0.23 \\
\pm 0.01\end{array}$ & $\begin{array}{l}0.23 \\
\pm 0.0\end{array}$ & $\begin{array}{c}0.21 \\
\pm 0.02\end{array}$ \\
\hline SFA & $\begin{array}{c}9.58 \\
\pm 0.24\end{array}$ & $\begin{array}{c}9.49 \\
\pm 0.35\end{array}$ & $\begin{array}{l}10.25 \\
\pm 0.41\end{array}$ & $\begin{array}{l}12.05 \\
\pm 0.52\end{array}$ & $\begin{array}{c}8.72 \\
\pm 0.26\end{array}$ & $\begin{array}{c}8.95 \pm 0 . \\
59\end{array}$ & $\begin{array}{c}10.67 \pm \\
0.33\end{array}$ & $\begin{array}{c}9.24 \\
\pm 0.22\end{array}$ & $\begin{array}{c}9.75 \\
\pm 0.31\end{array}$ & $\begin{array}{c}8.77 \\
\pm 0.40\end{array}$ & $\begin{array}{c}9.75 \\
\pm 0.28\end{array}$ & $\begin{array}{l}11.73 \\
\pm 0.37\end{array}$ & $\begin{array}{l}10.14 \\
\pm 0.54\end{array}$ \\
\hline MUFA & $\begin{array}{l}22.28 \\
\pm 0.34\end{array}$ & $\begin{array}{l}20.21 \\
\pm 0.23\end{array}$ & $\begin{array}{l}23.06 \\
\pm 0.27\end{array}$ & $\begin{array}{l}34.62 \\
\pm 0.54\end{array}$ & $\begin{array}{l}24.85 \\
\pm 1.39\end{array}$ & $\begin{array}{l}31.16 \\
\pm 1.24\end{array}$ & $\begin{array}{l}23.00 \\
\pm 1.55\end{array}$ & $\begin{array}{l}30.35 \\
\pm 1.03\end{array}$ & $\begin{array}{l}18.15 \\
\pm 0.30\end{array}$ & $\begin{array}{l}22.83 \\
\pm 0.72\end{array}$ & $\begin{array}{l}19.83 \\
\pm 1.01\end{array}$ & $\begin{array}{l}29.31 \\
\pm 1.35\end{array}$ & $\begin{array}{l}23.10 \\
\pm 1.18\end{array}$ \\
\hline PUFA & $\begin{array}{l}68.12 \\
\pm 2.72\end{array}$ & $\begin{array}{l}70.30 \\
\pm 2.03\end{array}$ & $\begin{array}{l}66.70 \\
\pm 3.05\end{array}$ & $\begin{array}{l}53.33 \\
\pm 1.68\end{array}$ & $\begin{array}{l}66.41 \\
\pm 2.39\end{array}$ & $\begin{array}{l}59.90 \\
\pm 3.57\end{array}$ & $\begin{array}{l}66.33 \\
\pm 2.51\end{array}$ & $\begin{array}{l}60.42 \\
\pm 2.20\end{array}$ & $\begin{array}{l}72.00 \\
\pm 2.61\end{array}$ & $\begin{array}{l}68.39 \\
\pm 3.21\end{array}$ & $\begin{array}{r}70.41 \\
\pm 2.66\end{array}$ & $\begin{array}{l}58.97 \\
\pm 2.88\end{array}$ & $\begin{array}{l}66.76 \\
\pm 1.90\end{array}$ \\
\hline $\begin{array}{l}\text { PUFA/ } \\
\text { MUFA }\end{array}$ & $\begin{array}{c}3.06 \\
\pm 0.08\end{array}$ & $\begin{array}{c}3.46 \\
\pm 0.06\end{array}$ & $\begin{array}{l}2.89 \\
\pm 0.1\end{array}$ & $\begin{array}{c}1.54 \\
\pm 0.02\end{array}$ & $\begin{array}{c}2.67 \\
\pm 0.05\end{array}$ & $\begin{array}{c}1.92 \\
\pm 0.04\end{array}$ & $\begin{array}{c}2.88 \\
\pm 0.09\end{array}$ & $\begin{array}{l}1.99 \\
\pm 0.0 \\
\end{array}$ & $\begin{array}{c}3.97 \\
\pm 0.09\end{array}$ & $\begin{array}{c}2.99 \\
\pm 0.05\end{array}$ & $\begin{array}{c}3.55 \\
\pm 0.05\end{array}$ & $\begin{array}{c}2.01 \\
\pm 0.01\end{array}$ & $\begin{array}{c}2.89 \\
\pm 0.07\end{array}$ \\
\hline
\end{tabular}

*Results are the mean value \pm standard deviation of three independent experiments; $* *$ Not detected

The differences between these findings and the findings of the present study involved linoleic (C18:2), oleic (C18:1) and linolenic (C18:3) acid. PUFA were the main group of fatty acids in walnut oil extracted from the cultivars studied, with values ranging from 53.33 to $72.00 \%$. The values for the MUFA group ranged from 18.15 to $34.62 \%$. SFA were a minor constituent, with values ranging from 8.72 to $12.05 \%$. 28 ȘK 850 had the lowest total MUFA content along with the highest PUFA content. It was also the cultivar with higher value of linoleic acid. 28 SK 925 had the lowest PUFA value and the highest MUFA value. It was the genotype having the highest value of oleic acid. These data were in general agreement with those of earlier reports (Amaral et al. 2003; 
Li et al. 2007; Venkatachalam and Sathe 2006; Pereira et al. 2008). The PUFA/MUFA ratio varied from 1.54 to 3.97 .

Martinez et al. (2006) reported that the PUFA/MUFA ratio changed from 2.22 to 4.54 in the walnut cultivars Franquette, Chandler and Criolla. In the current study, some walnut genotypes and cultivars contained higher amounts of oleic acid, linoleic acid, palmitic acid and linolenic acid than some other walnut genotypes. Walnut oil composition is affected by variety, geographical location, climatic effects, and treatment. For example, walnut oil composition is affected by the addition of fertiliser during growth. The composition of walnut oil is also affected by the maturity of the seed at harvest, its position on the plant, and its handling after harvest (Crews et al. 2005).The selected genotypes have nutritionally promising levels of minerals and fatty acids. The data reported in this paper confirmed that walnuts were a rich source of important nutrients that would be very beneficial to human health.

\section{REFERENCES}

Akca Y, Ozongun S. Selection of late leafing, late flowering, laterally fruitful walnut (Juglans regia L.) types in Turkey. N.Z. J. Crop Hort. Sci. 2004; 32: 337-342.

Akca Y, Sutyemez M, Ozgen M, Tuzen M, Mendil D. Determination of chemical properties of walnut (Juglans regia L.) cultivars grown in Turkey. Asian $J$ Chem. 2005; 17 (1): 548-552.

Amaral JS, Casal S, Pereira JA, Seabra RM, Oliveira BPP. Determination of sterol and fatty acid compositions, oxidative stability, and nutritional value of six walnut (Juglans regia L.) cultivars grown in Portugal. J. Agric. Food Chem. 2003; 51(26): 7698-7702.

AOAC Association of Official Agricultural Chemists. Official Methods of Analysis. 15th ed. Washington DC USA: AOAC; 1990.

AOCS Official Method Ce 2-66. The American Chemists Society. Champaign. Illinois, USA: 19921993.

Balta MF, Dogan A, Kazankaya A, Ozrenk K, Celik F. Pomological definition of native walnuts (Juglans regia L.) grown in central Bitlis. J. Biol Sci. 2007; 7(2): 442-444.
Celebioglu G, Ferhatoglu Y, Burak M. Selection and plantation walnuts in Turkey, In: The International Conference on Walnuts: Proceedings: 1988; Ataturk Central Horticultural Research Institute. YalovaTurkey, 1988. p. 83-87.

Charlot G, Germaine E. Le noyer: nouvelles techniques. Ed CTIFL, Paris: 1994.

Crews C, Hough P, Godward J, Brereton P, Lees M, Guiet $\mathrm{S}$, et al. Study of the main constituents of some authentic walnut oils. J. Agric. Food Chem. 2005; 53 (12): 4853-4860.

Çağlarırmak N. Biochemical and physical properties of some walnut genotypes (Juglans regia L.). Nahrung/Food. 2003; 47 (1): 28-32.

Garcia JM, Agar IT, Streif J. Lipid characterization in kernels from different walnut cultivars. Turk. J. Agric. For. 1994; 18 (3): 195-198.

Greve LC, Mcgranahan G, Hasey J, Snyder R, Kelly K, Goldhamer D, et al. Variation in polyunsaturated fatty acid composition of Persian walnut. J. Amer. Soc. Hort. Sci.1992; 117 (3): 518-522.

Grosso NR, Nepote V, Guzman CA. Chemical composition of some wild peanut species (Arachis L.) seeds. J. Agric. Food Chem. 2000; 48 (3): 806-809.

Korzeniowska J, Stanislawska- Glubiak E. Analysis of plant micronutrient composition using the Ane method, EJPAU. 2004; 7(2): \#02. Available from: http://www.ejpau.media.pl/volume7/issue2/agronomy /art-02.html

Lavedrine F, Ravel A, Villet A, Ducros V, Alary J. Mineral composition of two walnut cultivars originating in France and California. Food Chem. 2000; 68: 347-351.

Li L, Tsao R, Yang R, Kramer JKG, Hernandez M. Fatty acid profiles, tocopherol contents, and antioxidant activities of heartnut (Juglans ailanthiofolia Var. cordiformis) and Persian walnut (Juglans regia L.). J. Agric. Food Chem. 2007; 55 (4): 1164-1169.

Martinez ML, Mattea MA, Maestri DM. Varietal and crop year effects on lipid composition of walnut (Juglans regia L.) genotypes. J. Am. Oil Chem. Soc. 2006; 83 (9): 791-796.

Olez H. Researches on the modification of walnuts of Marmara (Juglans regia L.) using the selection method (in Turkish). J. Yalova Central Horticultural Research Institute. 1971; 4: 1-4.

Ozkan G, Koyuncu MA. Physical and chemical composition of some walnut (Juglans regia L) genotypes grown in Turkey. Grasas y Aceites. 2005; 56 (2): 141-146.

Paquat C, Houtfenne A.IUPAC, Standard methods for the analysis of oils, fats and derivatives. 7th ed. Oxford: Blackwell Scientific Publications; 1987. 
Pereira JA, Oliveira I, Sousa A, Ferreira ICFR, Bento A, Estevinho L. Bioactive properties and chemical composition of six walnut (Juglans regia L.) cultivars. Food Chem. Toxicol. 2008; 46: 21032111.

Prasad RBN. Walnuts and pecans. In: Encyclopedia of Food Science, Food Technol Nutr. London: Academic Press; 1994. p. 4828-4831.

Ruggeri S, Cappelloni M, Gambelli L, Nicoli S, Carnovale E. Chemical composition and nutritive value of nuts grown in Italy. Ital. J. Food Sci. 1998; 10: 243-252.

Savage GP. Chemical composition of walnuts (Juglans regia L.) grown in New Zealand. Plant Foods for Hum. Nutr. 2001; 56 (1): 75-82.

Souci SW, Fachmann W, Kraut H. Food composition and nutrition tables. Medpharm. Stuttgart: CRC Press; 1994.
Şahin İ, Akbaş H. Researches on the technological properties of walnuts gathered from different types and areas (in Turkish). First National Walnut Symposium of Turkey. 2001; Tokat-Turkey; 2001. p. 104-114.

Şen SM. Researches on the modification of walnuts of North-east Asia and East Blacksea regions using the selection method (in Turkish). Doğa Bilim Dergisi, 1983; 7: 163-170.

Venkatachalam M, Sathe SK. Chemical composition of selected edible nut seeds. J. Agric. Food Chem. 2006; 54 (13): 4705-4714.

Yarılgaç T, Koyuncu F, Koyuncu MA, Kazankaya A, Şen SM. Some promising walnut selections (Juglans regia L.). Acta Hort. 2001; 544: 93-96.

Zwarts L, Savage GP, McNeil DL. Fatty acid content of New Zealand-grown walnuts (Juglans regia L.). Int. J. Food Sci. Nutr. 1999; 50: 189-194. 


\section{PAGINA EM BRANCO}

